\title{
Emergent Color Categorization in a Neural Network trained for Object Recognition
}

\section{Authors:}

\author{
JP de Vries, A Akbarinia, A Flachot, KR Gegenfurtner
}

\begin{abstract}
Color is a prime example of categorical perception, yet it is still unclear why and how color categories emerge. The key questions revolve around to what extent perceptual and linguistic processes determine categories. While prelinguistic infants and animals appear to treat color categorically, several recent attempts to model category formation have successfully utilized communicative concepts to predict color categories. Considering this apparent discrepancy, we take a different approach. Rather than modeling categories directly, we focus on the potential emergence of color categories as the result of acquiring basic visual skills. For this, we investigated whether color is represented categorically in a convolutional neural network (CNN) trained to recognize objects in natural images. We systematically trained novel output layers to the CNN for a color classification task, and found that clear borders arise between novel (non-training) colors that are largely invariant to the training colors. We confirmed these border locations by searching for the optimal border placement using an evolutionary algorithm that relies on the principle of categorical perception. Our findings also extend to stimuli with multiple, colored, words of varying color contrast, as well as colored objects with larger colored surfaces. These results provide strong evidence that color categorization can emerge with the development of object recognition.
\end{abstract}

\section{Significance statement}

The origin of color categories has been a controversial topic for a long time, the key question being to what extent perceptual and linguistic processes determine categories. Typically, this has been evaluated by modeling the shape of categories directly. Here, we take a very different approach and evaluate categorization in a neural network built for recognizing objects in natural scenes. Interestingly, the network appears to build a categorical representation of color as a side effect of learning to classify objects. This shows categorization may be driven by acquiring basic visual skills, particularly those pertaining to objects. 


\section{Introduction}

Color vision is a prime example of categorical perception, and, as such, has received considerable attention across several research domains (Harnad, 1987). Being dependent on both linguistic and perceptual processing has made it difficult to pinpoint the mechanisms responsible for the emergence of color categories, and why particular colors are grouped into categories the way they are. This has led to a protracted debate as to what extent categorization develops universally (independent of local language and culture) and to what extent it is relative to local communication (for an elaborate discussion on the Sapir-Whorf hypothesis see Kay, 2015; Kay \& Kempton, 1984). Proponents of the universalist view have pointed toward the overlap in focal colors across different cultures (Regier, Kay, \& Cook, 2005). Furthermore, it appears that categories can emerge independent of language development: Pre-linguistic infants pay more attention to color changes crossing categorical borders than color changes within categories (e.g. Skelton, Catchpole, Abbott, Bosten, \& Franklin, 2017) and several animal species respond to color categorically (Caves et al., 2018; Jones, Osorio, \& Baddeley, 2001; Poralla \& Neumeyer, 2006). Relativists (e.g. Davidoff, 2001), however, point to the difficulty children have acquiring color names (Roberson, Davies, Davidoff, \& Shapiro, 2004) and the case of a patient whose language impairments were associated with color sorting problems (Roberson, Davidoff, \& Braisby, 1999). Also, while categorization has been found in birds and fish, researchers have failed to find categorization in some primates (e.g. baboons) and it has been argued that the methodology of other primate studies was biased towards finding categorical results (Fagot, Goldstein, Davidoff, \& Pickering, 2006). Moreover, just as universalists point to the strong commonality among the development of color categories, proponents of the relativist view highlight differences (e.g. Roberson, Davidoff, \& Davies, 2000; Roberson, Davidoff, Davies, \& Shapiro, 2005).

With the apparent contradictions in findings, in more recent years, the universalist versus relativist debate evolved from contrasting two extremes, to looking at how different factors contribute to the process of categorization (e.g. Kay \& Regier, 2006; Steels \& Belpaeme, 2005). Importantly, recent advances have also started taking into account the varying utility of colors (e.g. Conway, Ratnasingam, Jara-Ettinger, Futrell, \& Gibson, 2020; Gibson et al., 2017; Zaslavsky, Kemp, Tishby, \& Regier, 2019). One seminal finding is that, despite idiosyncrasies between cultures, overall, warm colors are communicated more efficiently than cool colors (Gibson et al., 2017). Subsequent papers have demonstrated that utilizing a perceptually uniform color space in combination with concepts from communication theory, such as an information bottleneck (Zaslavsky, Kemp, Regier, \& Tishby, 2018) or rate distortion (Twomey, Roberts, Brainard, \& Plotkin, 2020) can be powerful in modeling the shape of color categories. While the degree of importance for communication in shaping categories varies, many of these recent studies rely on communicative concepts when it comes to shaping color categories. Notably, a recent study where communicating deep neural networks played a discrimination game demonstrated that allowing continuous message passing made the emergent system more complex complex and decreased efficiency (Chaabouni, Kharitonov, Dupoux, \& Baroni, 2021).

While the modeling approaches incorporating communication principles have proven powerful in predicting categorization characteristics, the strong reliance on communication 
does not address the existence of what appears to be categorical behavior for color in the above-mentioned pre-linguistic infants and various animals. Also, a recent case study shows that color naming can be impaired while color categorization remains intact, emphasizing that in humans the link between communication and categorization can be decoupled (Siuda-Krzywicka et al., 2019). Furthermore, the trend at looking at the ecological relevance of color in shaping categories has also extended to animal research where it was found that host birds use a single-threshold decision rule in rejecting parasite eggs rather than the dissimilarity in color to their own eggs (Hanley et al., 2017) and female zebra finches categorically perceive the orange to red spectrum of male beak color (Caves et al., 2018). These latter findings particularly emphasize that while taking into account the variation in usefulness over colors through communicative concepts may be powerful, it does not preclude the possibility that the same usefulness variations can play a role during the acquisition of basic visual skill and, that this, by itself, could result in a categorical representation of color.

At the heart of these discussions lies the question of how and why color categories spread across perceptual color space, the way that they do. As described above, the possible causes range from visual perception being shaped based on the physical properties of the world all the way to the need for efficient communication about color. Focusing on color categorization as a potential emergent property of acquiring basic visual skills, here we show that a categorical representation of color can emerge automatically in a Convolutional Neural Network (CNN) trained to perform a basic object recognition task. With this, our approach is not to model specific color category data directly, nor to model specific brain processes. Rather, we research whether a categorical representation of color can emerge as a side effect of acquiring a different visual task. Previously, many studies on the representation of color in a CNN rely on physiological style approaches (Engilberge, Collins, \& Susstrunk, 2017; Flachot et al., 2020; Flachot \& Gegenfurtner, 2018; Rafegas \& Vanrell, 2018). Considering color categorization is likely a higher order process, we rely on classical principals from a long history of psychophysical studies to study the emergence of color categorization.

\section{Results}

Border Invariance. Perceptual research in non-human species requires indirect measures and match-to-sample tasks have successfully been utilized to study visual perception in numerous species for a long time (e.g. Kastak \& Schusterman, 1994; Skinner, 1950). Training pigeons to match colors to one of three main color samples, subsequently allowed Wright and Cumming (1971) to introduce novel colors to determine where the borders between colors lay for the pigeon. Repeating the experiment with different training colors, they found crossover points to be similar across experiments, indicating a categorical perception of color. Here we use a similar approach to evaluate the color representation of a Resnet18 CNN (He, Zhang, Ren, \& Sun, 2016) that has been trained on the ImageNet task categorizing objects occurring in natural images (Jia Deng et al., 2009). First (replacing the original output layer) a new classifier is trained on the network to classify stimuli containing a single word of a specific color (selected from narrow bands in the HSV color spectrum at maximum brightness and saturation, for stimulus examples see Figure $1 \mathrm{~A}$; the hue spectrum 
with corresponding training bands is depicted in 1B). In a second step we evaluate the network using colors from the whole hue spectrum and inspect to what classes the colors are generalized. As shown in Figure $1 \mathrm{C}$, colors from outside of the training bands are largely classified to the neighboring color bands. As a consequence, determining the borders between classes is straightforward (see Figure 1D). The important question in regard to categorization is what determines the locations of these borders? One option is that the borders are dependent on the positions of the training bands, meaning a shift in these bands should translate to a shift in borders. Alternatively, the borders between the colors stem from a categorical representation of color in the network. In this latter case we expect the borders to be (at least partially) invariant to shifts in the training bands. To investigate this, we repeated the above process many times while slightly shifting the training bands for each iteration. As we do not know how many categories to expect, we vary the number of output classes from 4 through 9 (note that of the 11 basic color terms, only 6 are present in the selected hue spectrum). The result for 6 output classes has been visualized in Figure $1 \mathrm{E}$. As the training bands are gradually shifted (as indicated by the black lines in Figure 1E) we find that the borders between categories appear largely invariant to these shifts in training bands.

A
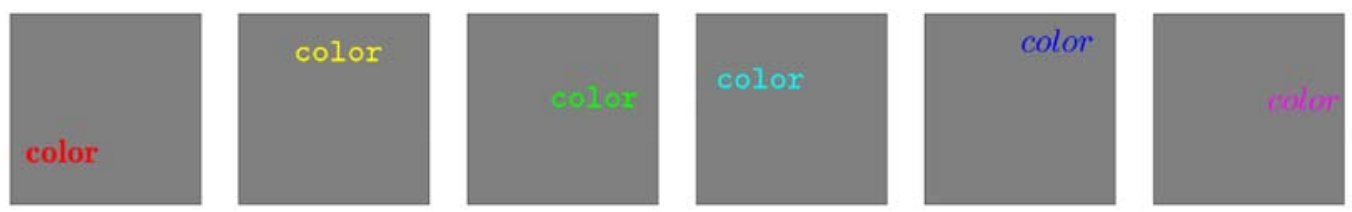

B

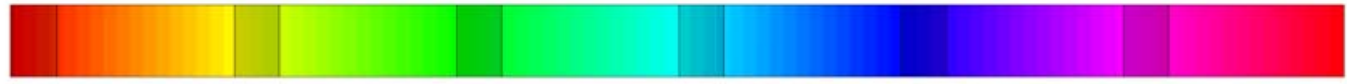

C
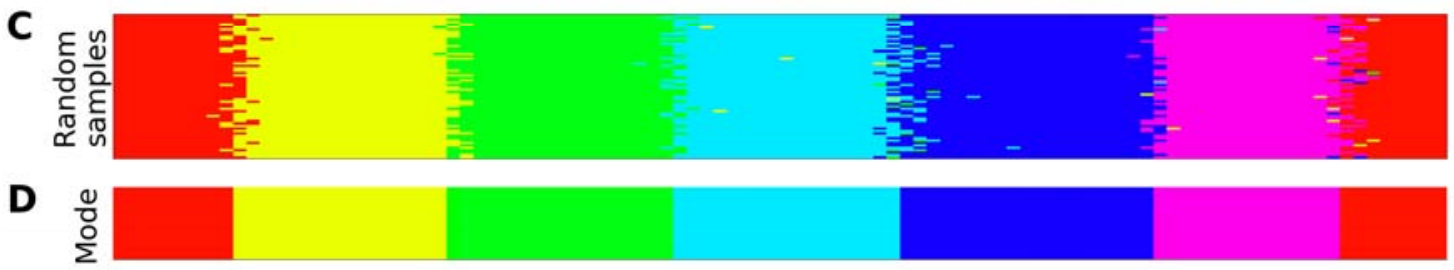

E

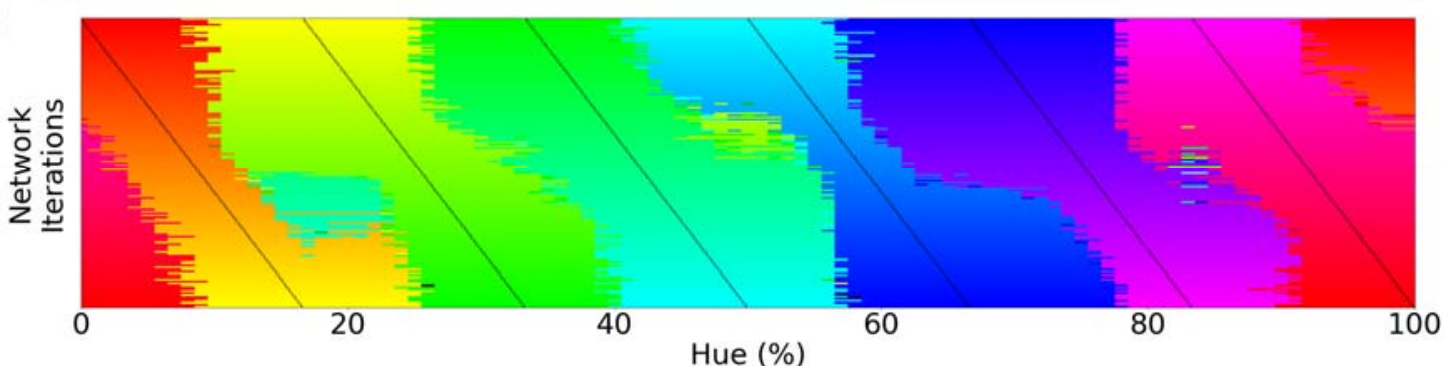

Figure 1. A) Six stimulus samples corresponding to the primary and secondary colors. B) Hue spectrum from HSV color space (at maximum brightness and saturation). The colors for each class are selected from narrow, uniformly distributed, bands over the hue spectrum. Bands are indicated by the transparent rectangles. C) Results from an individual training iteration for the bands depicted in $\mathbf{B}$. In each iteration the same ImageNet-trained Resnet-18 is used, but a novel classifier is trained to perform the color classification task with the number of output nodes corresponding to the number of training bands. Each pixel represents a classified sample, colored for the class it has been assigned to (based on the hue of the center of the training band). D) A one-dimensional color signal produced by taking the mode of each column in C. In this manner we obtain an overall prediction for each point on the spectrum and can determine where the borders between classes occur. E) Results for networks trained on 6 bands on the 
hue spectrum. Each row represents the classification of a single network (as in D), trained on 6 bands, the center of which is marked by a black tick (appearing as black diagonal lines throughout the image).

To determine whether the borders are consistent across the shifting training bands, we plot the transition count that indicates the co-occurrence of borders in Figure $2 \mathrm{~A}$ (i.e., the number of times a border occurs in a specific location, while the bands are shifted). Note that while Figure 1E only displays the results for a network trained with 6 output nodes, the full result depends on borders found when training the network with 4, 5, 6, 7, 8 or 9 output nodes. As those results are collapsed, we see that the transition counts seem to form about 7 to 8 discontinuities in the hue circle. Using a simple peak detection algorithm that relies on neighboring data points to find peaks, 7 peaks are found (red dots in Figure 2A). Utilizing those peaks, we divide the color in 7 different regions (Figure $2 \mathrm{C}$ ), and averaging the colors in each region (weighing them based on the reciprocal of the transition count) results in 7 colors that can be seen as representing each category.
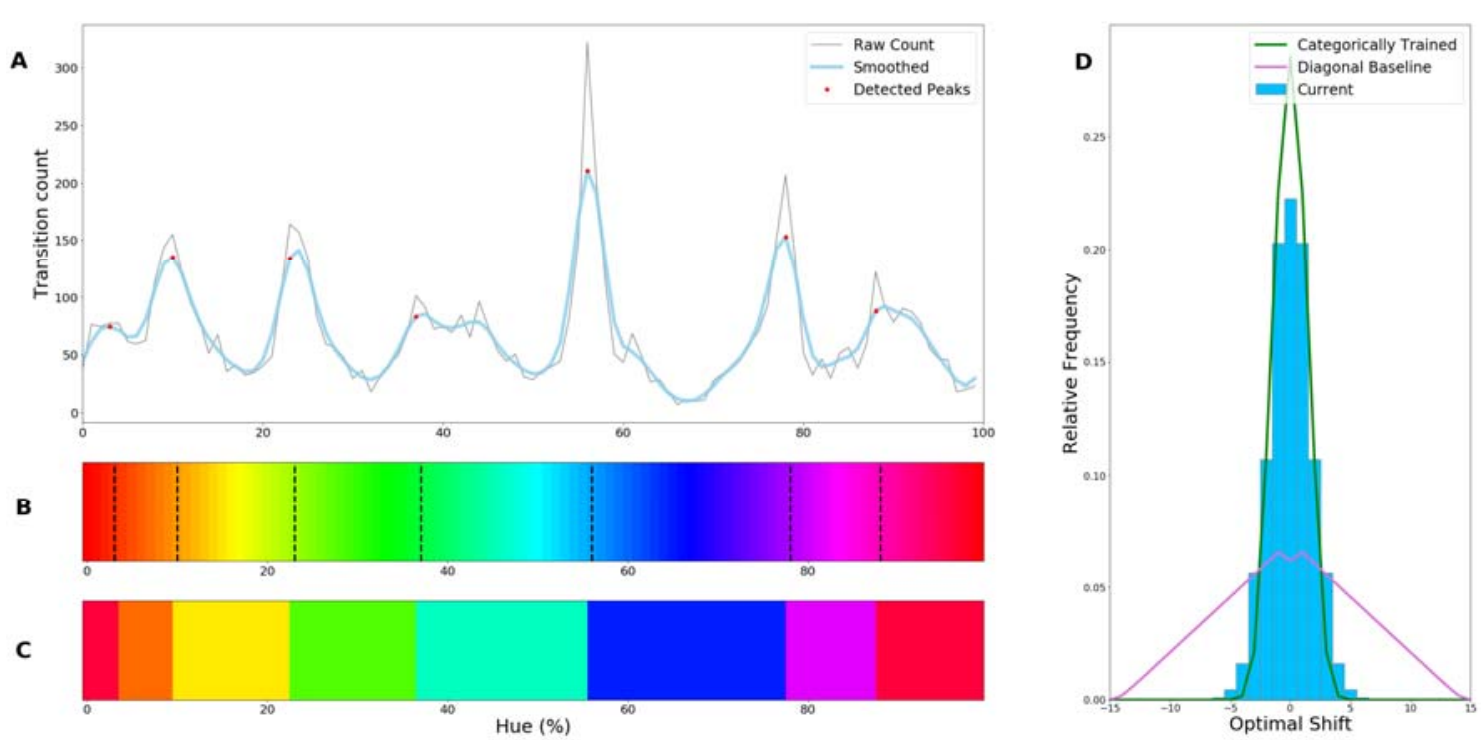

Figure 2. Border transitions in the color classifications. A) Summation of border transitions, calculated by counting the border transitions (as depicted in Figure 1D and E) for each point on the HSV hue spectrum (thin grey line). A smoothed signal (using a Gaussian kernel; blue thick line) is plotted to reduce the noise. Peaks in the signal (raw count) are found using a simple peak detection algorithm (argrelextrema from the scipy.signal library) and indicated in red. B) The peaks superimposed on the hue spectrum as vertical black dotted lines. C) Category prototypes for each color class obtained by averaging the color in between the two borders (using reciprocal weighing of the raw transition count in A. D) For each row (as in Figure 1E) the optimal cross correlation is found by comparing each row to every other row and shifting it to obtain the maximum correlation. In blue we plot the distribution of shifts for the when 7 output classes are used (as we appear to find 7 categories). For comparison we plot the result of a borderless situation (where borders shift with training bands) in purple and in green the result for a network trained from scratch on our 7 different color categories.

Considering the degree of invariance is not constant over the different borders, the question becomes what we consider sufficient evidence for finding borders. Naturally, if the color representation would be strictly categorical, the result in Figure $1 \mathrm{E}$ would primarily show straight lines. On the other hand, if the network incorporated some form a continuous representation of color, we would expect borders to be shifting with the shifting color bands and diagonal transitions, following the diagonal of the training bands, would be most prominent. In the first case, the highest cross correlation between rows would be found by 
keeping them in place as is. However, in the latter case, the maximum cross-correlation would be found by shifting the rows with respect to each other row. We can contrast the two cases by calculating, for each pair of rows, the lateral shift that produces the highest cross correlation and inspect the resulting distributions. If the borders are stable across the different training iterations, the distribution of such shifts to obtain the maximum cross correlations would be centered narrowly around zero. If the borders move along with the training colors, the distribution would be much wider. Simulations for these two particular cases are shown by the green and purple curve in Figure $2 \mathrm{E}$, respectively. The histogram plotted in light blue shows the actual data, which more closely follow the green distribution, representing the categorical simulation. The difference is substantiated by a K-Sample Anderson-Darling test that is significantly different when comparing the found distribution with the resulting distribution for the continuous case (stat=7.1; $p<0.001$ ), but not when being compared to the categorical case (stat $=0.8 ; p=0.15$ ).

We have elected to use the hue spectrum from the HSV color space as it includes multiple colors belonging to the basic color terms and because neighboring colors in HSV will be neighboring colors in the RGB space (in which our network was trained) at a constant distance. Varying the hue in the HSV space, however, does not only change the color of the word stimuli, but also their luminance. To ensure the current borders do not stem from a spurious correlation between color and luminance we have rerun the current experiment with different stimuli that include luminance distractors and a variable background luminance (see SI:Luminance Variation). While border locations are not a perfect one-to-one match with the current results, overall, they do seem similar and a categorical representation is again found. With the introduction of distractors and variation in background luminance the network can rely only on kernels coding purely for color and not a combination of color and luminance to perform the task. We also explored an alternative hue spectrum from a single plane of RGB color space. This led again to a categorical representation, albeit more noisy, presumably due to the reduction in chromatic contrast (see SI: Circular Color Spectrum).

Evolutionary algorithm using principles of categorical perception. The generalization over neighboring colors and the borders between them are consistent with the notion that the network builds a categorical representation of color. Nevertheless, the lack of a broad understanding of CNNs in general and particularly their representation of color makes us wary to draw a definitive conclusion from only the first experiment. To evaluate whether the colors within the boundaries can indeed best be seen as belonging to categories we turn to the concept of categorical perception, where differences between colors within the same category are seen as smaller than differences between colors from different categories (e.g. Goldstone \& Hendrickson, 2010). If the discontinuities we found indeed mark borders in a categorical representation of color, we expect that generalizing colors between 2 discontinuities should be easier than generalizing colors that cross discontinuities. In humans, categorical perception is often studied using reaction time tasks (e.g. Paul Kay, Regier, Gilbert, \& Ivry, 2009; Winawer et al., 2007; Witzel \& Gegenfurtner, 2011), but a direct analogue of reaction times in neural networks is not available. There is however a different temporal performance measure available: the ability to evaluate how quickly a network can learn a task. If the boundaries found in the first experiment indeed resemble categorical borders, it should be faster for the network to learn to generalize colors within 
two neighboring discontinuities to a specific class than to generalize colors crossing discontinuities.

Specifically, in the current experiment we evaluate how well a set of borders fits the color representation of the network by evaluating how easily sets of 2 narrow training bands placed directly inside of these borders can be generalized to single classes. One straightforward way of evaluating the discontinuities found above would be to make direct comparisons based on their locations. However, this would either mean using biased comparisons based on the borders found above, or require an almost infinite number of comparisons to ensure we compare the found borders to every alternative. To avoid this, rather than using the previously found borders as a starting point, we developed a search algorithm that uses the principle of categorical perception to find the optimal set of borders for the network from scratch. The only information taken into account from the previous experiment is that approximately 7 borders were found: We employ an evolutionary algorithm that starts with 100 sets of 7 randomly initialized borders. Allowing the network to train for a limited number of epochs (the number of times the network sees every sample in the training set) we evaluate the fitness of each of the randomly initialized border sets. Using the fitness of each set, the best 10 performers are copied to the next generation (elitism) as well a set of 90 novel border instantiations. The latter instantiations are generated by randomly selecting parents from the previous generation (with a bias for better performing ones) and recombining their borders to create new border sets. By allowing this process to run for some 40 generations it converges to a specific set of borders with little variation between the top ten performers. As evolutionary algorithms are not guaranteed to converge to a global optimum, we ran the algorithm 12 times to ensure the results are consistent. In the current case the borders should be less variable, but still line up with the peaks of the invariant border experiment.

Figure 3 shows where the evolutionary algorithm places the borders and we see a strong correspondence with the previously found borders (indicated by black vertical dotted lines). The only exception appears in the border between green and turquoise. However, note that this is also the region of color space where the transition count (as shown in 2A) did not show a sharp peak, as was the case for most other borders. Interestingly, this is also the border where the largest variability is observed for human observers (Hansen \& Gegenfurtner, 2017; Figure 2E). The current results suggest that the best explanation for the discontinuities we observed in the first experiment are that they indeed represent categorical borders dividing the color space. The fact that color categorization emerges in a CNN trained for object recognition underscores that color categorization may be important to recognizing elements in our visual world (see Witzel \& Gegenfurtner, 2018). This would also explain why there are strong universal tendencies in the development of color categories across cultures (e.g. Kay \& Regier, 2003). Further exploring the origin of the borders, the results shown in SI: k-means clustering indicate that the distribution of colors in the ImageNet database within the hue spectrum we used might, in part, explain the borders, in line with earlier results by Yendrikhovskij (2001). 


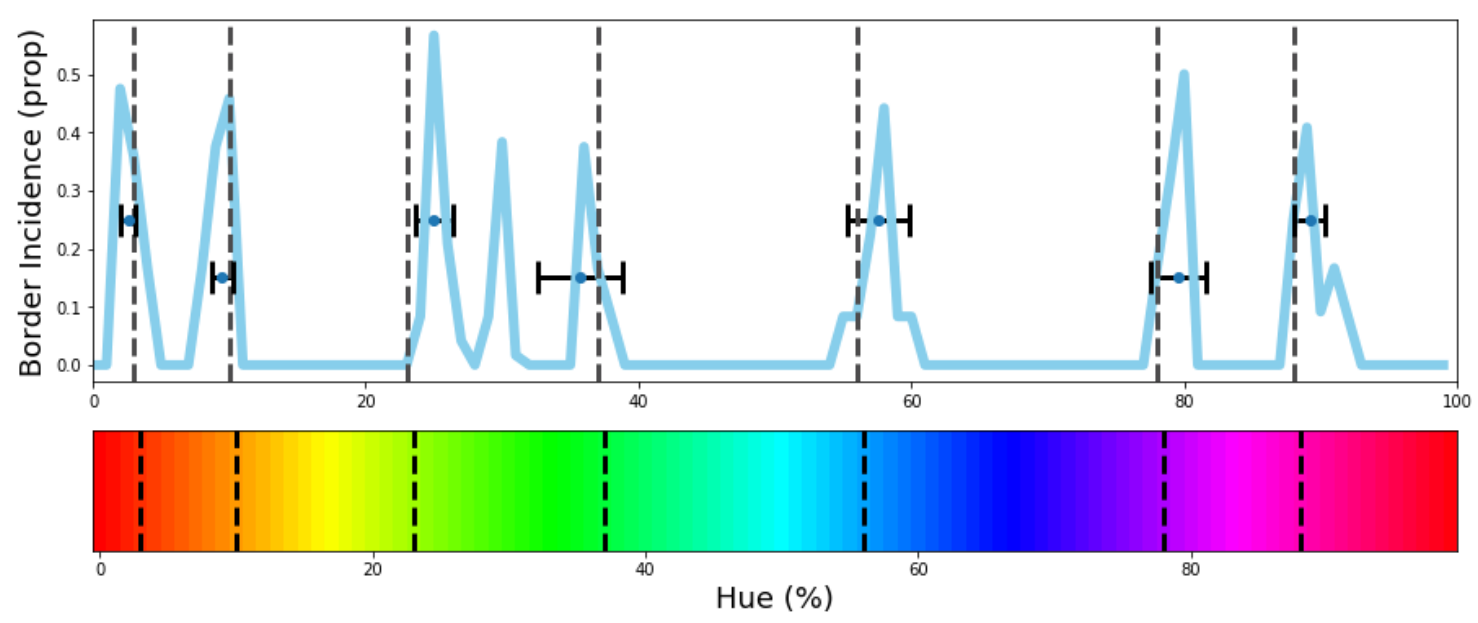

Figure 3. The evolutionary algorithm is repeated 12 times and we calculate the frequency of borders for in each of the top 10 border sets. The resulting frequencies are plotted in blue. Border location estimates from the invariant border experiment are plotted in the graph and on the hue spectrum in dotted black vertical lines for comparison. Ordering the borders in each solution from left to right allows us to derive an estimate for each border the evolutionary algorithm produces by taking the median for the first, second etc. values, respectively. We have plotted these medians as points, including a horizontal errorbar that indicates a standard deviation to demonstrate the variability for these values. As can be seen, the estimate for each column agrees closely to the estimate from the invariant border experiment.

Complex color stimuli. The converging results from the above experiments provide a strong indication that the network represents colors categorically. Still, the stimuli used above deviate considerably from the ImageNet database. We, therefore, wanted to ensure that the found borders are directly connected to how the network deals with color. We proceed by investigating to what extent the borders generalize to tasks more similar to those the network was originally trained on and introduce a more complex set of stimuli that are comprised of multiple, colored, words on a randomly colored background to introduce contrast variations (see Figure 4A). Specifically, stimuli were comprised of 3 words colored based on the training specifications, as well as 2 additional words that were colored randomly. Finally, the background color is also randomly selected from the hue spectrum, however, at a lower brightness to ensure that there is sufficient contrast between words and background.

The key question is not whether the network can perform this task, but whether the obtained categories are meaningful in light of more complex color images. Therefore, we trained novel output layers while iteratively shifting training bands from left to right within each category (in 10 steps). This allows us to evaluate performance as a function of the training band positions within the category. Two possible outcomes can be distinguished: On the one hand it is possible that for such complex stimuli the network deviates from the obtained categories and performance does not depend on where we select our training bands in the category. Alternatively, if the system can benefit from the categorical coding of color, we expect performance to be highest and the error rate to be lowest at the center of the categories, while the error rate should peak when the training bands align with the borders of the categories. In Figure 4 we see that this latter categorical representation is indeed what the network relies on: Overall, the network is able to perform the task reasonably well, but error rates are lowest towards the category centers, while increasing 
towards the borders. As in the experiments above, there is a slight divergence at border between green and turquoise.
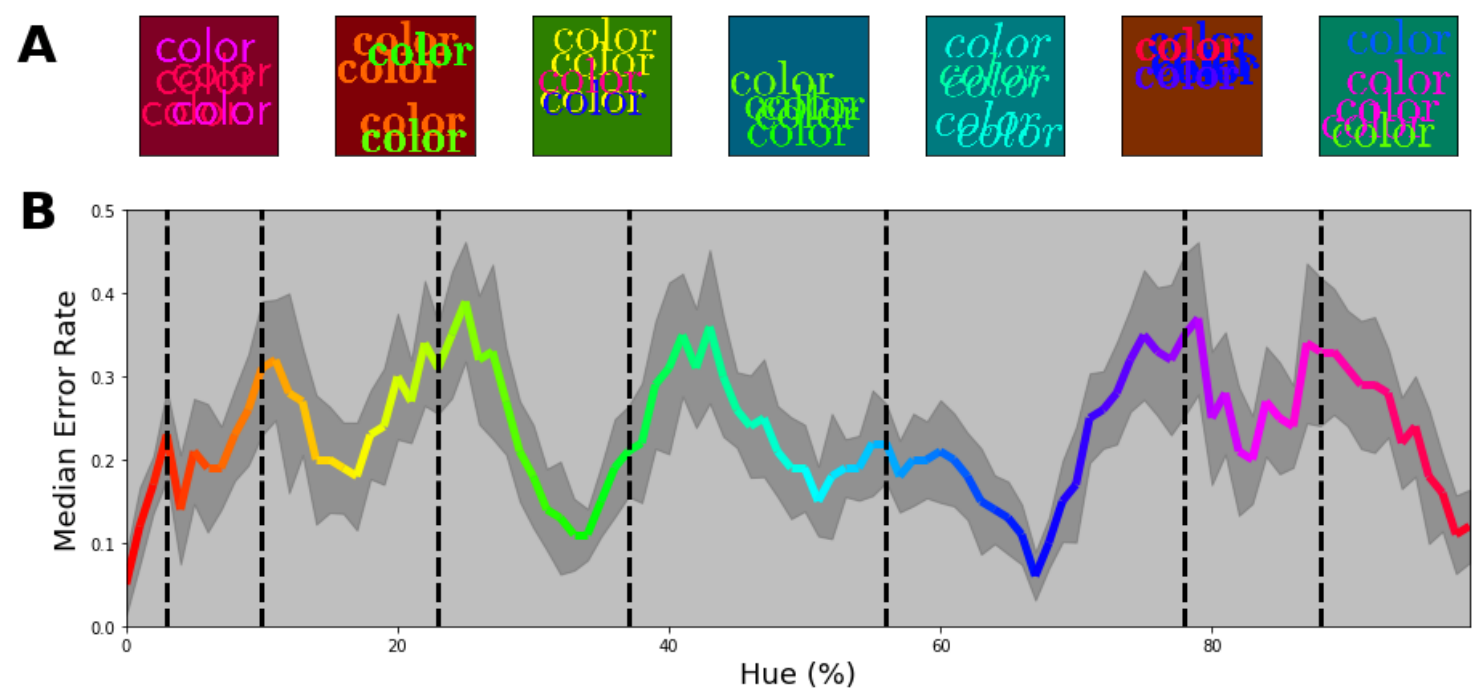

Figure 4. Multi-colored stimuli classification performance. A) 7 example stimuli, each sampled from a different color band. Each stimulus consists of 3 equally colored (target) words of which the color is determined by the selected class. Subsequently, 2 randomly colored (distractor) words, also randomly positioned, were drawn on top. These words are randomly positioned in the image. Finally, the background for each image is chosen randomly from the spectrum, but with a reduced brightness of $50 \%$. B) Error proportion as a function of hue. Separate output layers have been trained on color bands that are shifted from the left to the right border in 10 steps for each category at the same time. This means, that while one network is trained to classify words of colors sampled from a narrow range on the left side of each category, another network is trained to classify words of colors sampled from the right side of each category for each respective class. After training the performance is measured using novel samples on the hue spectrum that match the color bands the network is trained is. Subsequently, the resulting error rate is displayed in the colored line by combining the performance for all the networks (shaded grey region represents one standard deviation). In this manner we can see the error rate typically increases as it approaches a border. 
Recognizing colored objects. The previous experiment extends our finding to more complex color stimuli. We chose word-stimuli because they are made up of a rich set of patterns with many orientations. One notable element missing in these word stimuli are the large surface areas that are typically found in objects. In this experiment we investigated whether the previously found categorical borders still guide classification when classifying objects that incorporate large uniform areas. For generating objects with larger uniformly colored areas we rely on the Google Doodle Dataset (Ha \& Eck, 2017). This dataset includes thousands of examples of hand drawn objects, gathered from individual users. Because each drawing in the dataset is stored as a series of vectors it lends itself well to redraw the lines and fill the resulting shape with a uniform color (we plot some examples in Figure 5A). To further evaluate the usage of color categories in object recognition of the CNN we added one additional manipulation. So far, our experiments have aimed at looking on the reliance on color in isolation of potential other factors. However, with the introduction of objects of different shapes, a natural question is to what extent the network uses color or shape to classify the objects? To obtain a better insight into the interaction between these components we raised the number of classes from 7 to 14 . This allows us to evaluate whether the network simply ignores the color categories when they are not the sole source of discrimination, or can use them in combination with shape features.

We ran several iterations, selecting random permutations of 14 objects for the 14 training bands on the HSV hue spectrum (2 per category). In Figure 5B the 14 training bands are indicated using transparent, colored, bars, while the black dotted vertical lines again indicate category borders as obtained in the invariant border experiment. The proportion correct, plotted in the color of the hue for each object is measured over the whole hue spectrum (based on 100 permutations reassigning different objects to the colored bands) and the grey shaded area indicates one standard deviation. We observe that performance is consistently high within the category borders in which the training band falls, while in most cases there is a steep drop in performance outside of the category bounds. This means that on the one hand the network uses color to distinguish objects from those that are colored for different categories. At the same time, however, it appears that to discern two objects within a category, classification relies on the object shape. As such, the network appears to combine both color and shape information and, important to the current research question, the representation of color it relies on, closely follows the previously found category borders. 
A
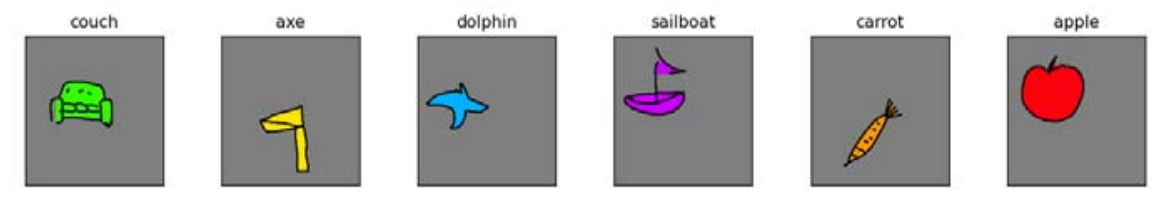

B

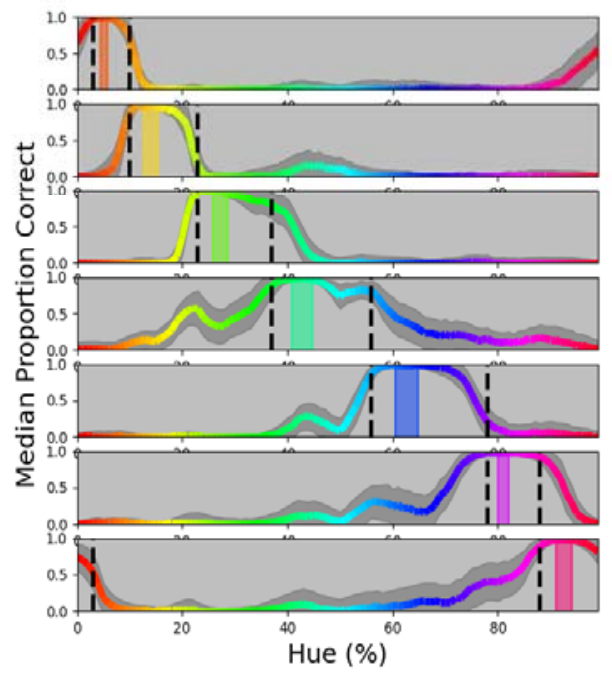

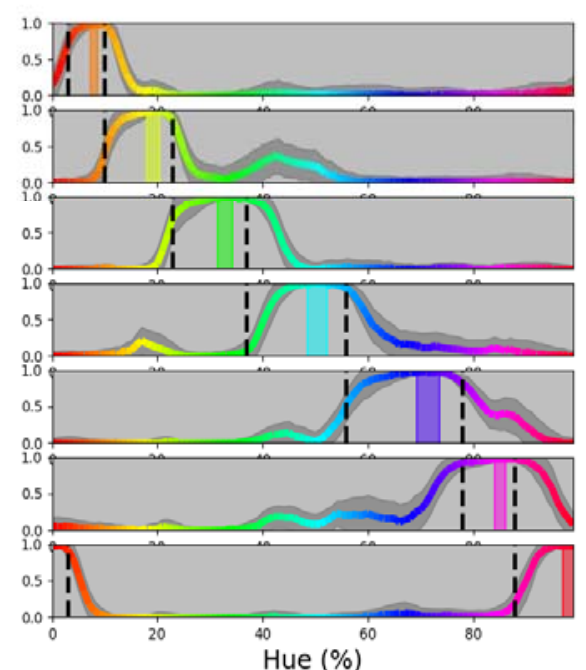

Figure 5. A) Samples of the google doodle dataset as colored by our simple coloring algorithm. B) Proportion correct as a function of hue. The 14 individual plots correspond to the 14 training bands that have been selected, 2 per category, 1 to the left of the category center, one to the right. The training bands are indicated by transparent rectangles on the spectrum, colored for the center of the band. The colored line represents the median proportion correct for the network on samples of the respective hue. Objects corresponding to each training band were randomized and the calculation was repeated 100 times. Per instance, 80 drawings of the respective object are filled with each color on the hue (in 100 steps) and proportion correct has been calculated for each instance and hue individually. Lines correspond to the median proportion correct over the 100 repetitions. The shaded grey area indicates the standard deviation over the 100 repetitions.

\section{Discussion}

A number of recent studies have focused on the development of categories by explicitly modeling color categorization. Here we have taken a different approach by evaluating whether color categorization could be a side effect of acquiring a basic visual skill. We found that color categorization is an emergent property of a Convolutional Neural Network (CNN) trained for object recognition. Importantly, our findings are able to unite much of the previous research on color categorization. First, they can explain why the emergence of color categories over cultures broadly follows a universal pattern. Second, the findings are in line with the notion that color categorization emerges in pre-linguistic infants and animals. Third, the findings are in line with recent reports showing a dissociation between color naming and color categorization. Fourth, while the findings may seem in contradiction to recent models relying strongly on communicative concepts, the varying communicative need these models rely on, likely correlates with the varying utility of colors to more basic visual tasks, particularly, those pertaining to objects. As such, the predictive power of these models may not rely on communication about colors per se, but on taking into account the 
varying utility over colors. Therefore, it is likely that the implicit incorporation of color patterns useful during object recognition leads to a categorical representation of color in a CNN.

Originally, categorization was theorized to be culturally dependent (e.g. Ray, 1952). However, in 1969 Berlin and Kay proposed that color categories across a wide range of languages could be described using 11 basic categories, as such putting forth that a universal process guides the development of categories across cultures. While this notion was first hypothesized based on visual inspection of collected categorization data, later, evidence was provided by demonstrating statistical regularities across cultures (Kay \& Regier, 2003; Regier et al., 2005). The current findings can explain why the general development of categories is so similar across languages: If color categorization is a side effect of acquiring basic visual skills, given relatively similar circumstances across the globe, color categories are expected to shape in a similar fashion throughout many cultures. Naturally, this does not preclude local differences in the visual surroundings and communicative need from further shaping categories, however, the current findings do offer a potential cause for the broad similarities in color categories and their development.

Measuring the full hue circle in pre-linguistic infants, Skelton and colleagues (2017) found that the typical novelty preference was dependent on categorical crossings. As such it appears that some form of categorical representation develops early on. However, it is unclear whether these categories are the direct basis for those that develop later in life. Four of the five categorical distinctions Skelton and colleagues found can be separated by the cardinal axis corresponding to the color representation in the retinogeniculate pathways, suggesting some of the categorical behavior may rely on the early representation of color (this issue has also been pointed out by, e.g. Lindsey et al., 2010; Witzel \& Gegenfurtner, 2013). Also, color naming develops much later and children that have not yet acquired color names make recognition errors based on perceptual difference (Roberson et al., 2004). Similarly, it is unclear whether categorical representations of color in animals resemble those in humans. Nevertheless, recent findings have shown, that, as in humans, the utility of color may play an important role for the categorical color representation in animals (Caves et al., 2018; Hanley et al., 2017). As such, while the nature of the categorical representations in prelinguistic infants and animals is still somewhat unclear, the novel finding that a categorical perception emerges with the general acquisition of visual skills is in line with the current findings.

Similar to the approach in animals, we relied on a match-to-sample task for studying color categories in a CNN. With this indirect approach, some limitations are similar to those in studies on animals. Importantly, however, there are also clear advantages to studying categorization in a CNN. We were able to repeat the match-to-sample task for a great number of training colors, without risking a bias over training sessions allowing for better estimates. Moreover, verifying the borders using the concept of categorical perception with an evolutionary algorithm is a computationally intensive task that cannot be straightforwardly applied to any living system, but is feasible for CNNs because of the speed at which an output layer can be retrained. Of course, compared to animal and infant models the CNN is the least convincing version of the adult visual system. Nevertheless, an important benefit is that as the access to activity of artificial neurons is complete it can be 
exploited in future research: While neural networks are often described as black boxes, compared to biological systems, artificial neural activity is much more accessible, as one can easily probe the activation of all neurons. With this kind of access, a logical next step is to investigate how the obtained categories are coded in the CNN. In humans, coding of colors seems to become narrower and more variable beyond the LGN (Kiper, Fenstemaker, \& Gegenfurtner, 1997; Lennie, Krauskopf, \& Sclar, 1990) and colors belonging to the same category seem to be clustered together (Brouwer \& Heeger, 2013). Zaidi and Conway (2019) suggested such narrowing may take place over areas (from V1 to IT) through operations equivalent to a logical AND or clustering. The CNN can serve as an important model for testing the viability of these concepts.

Where the terms color categorization and color naming are often used synonymously, the subtle distinction between them is key to the debate on the emergence of color categorization. From a strong universalist point of view, a color name is no more than a label to categories that have developed perceptually. From the relativist point of view, the direction is reversed and it is the existence of a color term that dictates the existence of the respective category (e.g. Jraissati, 2014). A recent case study shows a dissociation between color naming and categorization. Patient RDS is able to categorize colors, but his color naming ability is impaired (Siuda-Krzywicka et al., 2019, 2020). The dissociation between the two, favor a view where categorization is a process that can exist independently of the linguistical labels. Interestingly, despite the problems in color naming, the link between objects and colors was preserved: patient RDS would often use this link to perform color naming tasks: "this is the color of blood; it must be red" (page 2473; Siuda-Krzywicka et al., 2019). While it is possible to argue that our CNN does communicate (it assigns objects to a class), it is important to note that our network at no point is required to communicate about colors directly, but at best about objects. As such, the current finding effectively lines up with the notion that colors and their categories may be formed as part of a way to identify objects.

The fact that the current categorical representation appears to emerge in the absence of color naming shows that explicit color naming is not a necessity for the development of categories. This may seem to stand in contrast to many of the recent studies that use communicative concepts as a means to model the shape of categories (Chaabouni et al., 2021; Gibson et al., 2017; Twomey et al., 2020; Zaslavsky, Kemp, Tishby, \& Regier, 2020). However, many of those studies derive their predictive power from combining these communicative concepts with the non-uniformities in the utility across colors. As such, the communicative concepts could just be a means to incorporate the variation in utility across colors. For instance, where it has been demonstrated that warmer colors are communicated more efficiently than cooler colors (Gibson et al., 2017), it has also been shown that objects are associated with warmer colors than backgrounds (Rosenthal et al., 2018). The latter emphasizes that the higher communicative need for warmer colors likely stems from their prevalence in objects. While we do not argue the process is devoid from communicative factors, the current results can unify the previous findings by showing that acquiring a skill like object recognition can lead to the emergence of a categorical representation of color. 


\section{Methods}

\section{Invariant Border Experiment}

\section{Software architecture and Stimuli}

The experiment utilizes a Resnet-18 as provided in the models module from the torchvision package (Marcel \& Rodriguez, 2010). The network is initialized with the pretrained option on: weights are set based on having been trained on ImageNet (Jia Deng et al., 2009). After initializing the network, we replace the output layer for object recognition with a smaller output layer with anywhere from 4 to 9 output nodes. The weights to this novel (replacement) classification layer are randomly initialized.

Stimulus images are generated using the Pillow package (Clark, 2015). Image size is the same as that used for the original ImageNet training (224×224 pixels). Each image contains the word "color" randomly positioned on a mid-grey background (font size 40, approximately 100 by 25 pixels, depending on font type). The color of the word is randomly (uniform) selected from the bands on the hue spectrum in HSV space. Color bands representing the individual classes are uniformly distributed over the hue space (see Figure $1 \mathrm{~A}$ for stimuli sampled from example bands in Figure 1B). Brightness and saturation are set to the maximum level. The HSV color for each pixel is converted to its equivalent in RGB space as the network has been trained using three input channels for red, green and blue, respectively. The font of the word is selected from 5 different fonts.

\section{Procedure}

For each number of output nodes (4 through 9), we initialize 150 versions of the network and train each on a slightly shifted set of training bands. The combined width of the training bands equals $20 \%$ of the total hue range. During network training we only allow the weights of the novel classifier to be updated, the weights of all preceding layers remain as they were trained on ImageNet. Each novel output layer is trained on a slightly shifted set of hue bands. Because we cannot determine the number of potential color categories a-priori, we vary the number of output classes from now 4 through 9 . This results in training a newly initialized output layer for 150 (band shifts) times 6 (4 through 9 output classes) networks, making for a total of 900 training sessions. 500 samples are provided for each class and the network is trained for 5 epochs. During the training we keep track of the best network using 50 separate validation samples per class (from the same training bands). After training the network to classify the colors from the training bands, each network is evaluated over the whole hue spectrum by providing the network with 60 samples for each step on the hue spectrum (divided into 100 steps). This results in 6000 classified samples for each of the 900 trained networks.

\section{Analysis}

The 6000 test samples for each trained output layer are used to determine the border crossings. In Figure $1 \mathrm{C}$ we plot the classification of these 6000 samples for a single training iteration. To determine the best prediction, for each step on the hue spectrum (each column in Figure $1 \mathrm{C}$ ), we take the mode. In this manner we transition to a one-dimensional representation of the network's performance on the evaluation task, with the prediction for each hue. Importantly, this one-dimensional representation, as plotted in Figure 1D, is used to determine the border crossings: for each network we determine the borders by simply 
picking the transition between predicted classes. Finally, we sum all the borders and from this we use a straightforward peak detection algorithm to find the local spots on the spectrum where the borders are most invariant to change. To determine how "categorical" the found border invariances are, we determine the maximum cross-correlation for each row, compared to every other row, by shifting one of the rows and finding the optimal shift by looking for the maximum cross correlation. To maintain the circular nature, rather than the ordinal colors, a semi sine wave conversion is applied to the predicted hues. By obtaining the shift for each row, with all other rows, we obtain a distribution of shifts, that can be compared to distributions representing a categorical result and continuous color result. The former is generated by training a Resnet-18 (from scratch) on the currently obtained categories and, subsequently, evaluating it in the same manner as we evaluated the Resnet-18 trained on ImageNet. The latter distribution is determined by calculating the optimal shift for the case where the borders between colors move in parallel to the shifting bands.

\section{Evolutionary Experiment}

Stimuli are the same as the previous experiment. We initialize 100 sets of 7 borders, each ordered from left to right (each representing a position on the hue spectrum). For each border set a Resnet-18 pre-trained on ImageNet is initiated and again the final fully connected layer is replaced. The stimuli for training the networks are generated via the same procedure as described in the Invariant Border Experiment, but now the hues for each class are selected from two narrow bands just inside of each set of neighboring borders (see Figure 6). Both band positions, as well as width, are relative to the two adjacent borders; The band starts slightly inside the border (with the closest edge located $5 \%$ from the border) and the band width is set to $10 \%$ of the total distance between the borders. We use two narrow bands at the ends of the potential category as this will mean that when the borders cross a categorical boundary the network will have to learn to generalize colors from different categories to single classes, while if the borders are located optimally, the 2 bands for each class will stem from 1 category each. We judge the fitness of a border set by evaluating how fast the network can learn the classes defined by the bands on the inside of two adjacent borders. Therefore, each network is trained for 3 epochs only, which is insufficient to reach peak performance, but allows us to evaluate which border set best fits the color representation of our Resnet: Border sets that align with the color representation of the network should allow the network to reach a higher performance quicker.

B1

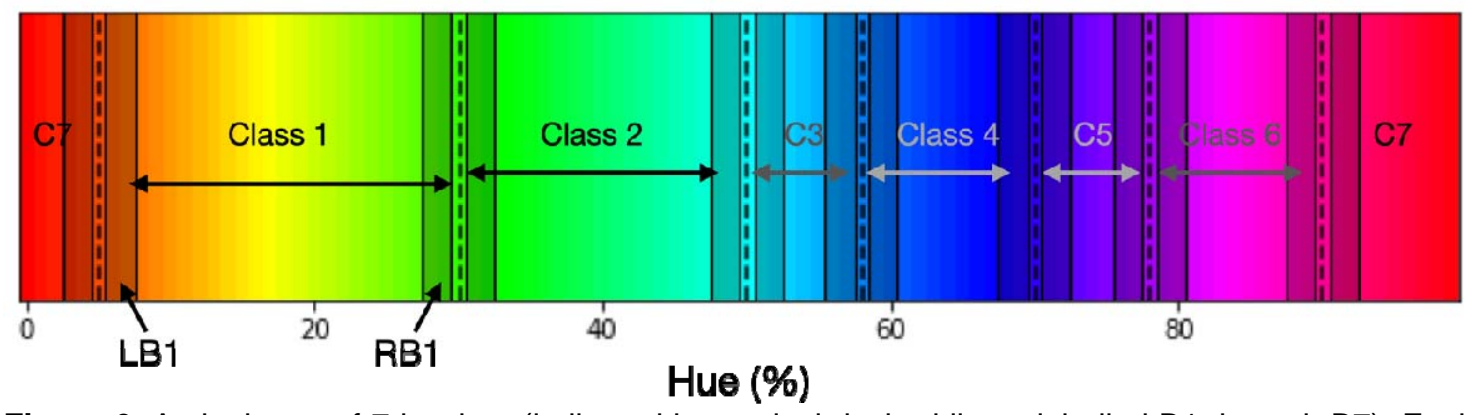

Figure 6. A single set of 7 borders (indicated by vertical dashed lines; labelled B1 through B7). Each space in between two adjacent borders represents a class. Colors for the training samples for, for 
example, Class 1 are randomly selected from one of the two bands, LB1 (Left Band for Class 1) and RB1 (Right Band for Class 1), on the inside of the borders of the class. Each of the two bands (not drawn to scale) comprises $10 \%$ of the space between; for class $\mathrm{C} 1$ this is the distance between first dotted vertical line (B1) and the second dotted vertical line (B2). Note that this means that the bands for class C3 for example are thinner than for Class 1. To ensure the training bands do not overlap at the borders, there is a gap (comprising $5 \%$ of the category space) between the border and the start of the band.

After training the 100 networks, the border sets are sorted by the performance of their respective network. Note that while we base the ordering on the performance of the network, the network is assumed a constant factor and the performance is attributed to the border set that is used to generate the training set; Essentially the network is the fitness function for the border sets. Once the fitness of each border representation has been established, the sets can be ordered by fitness and we generate a new generation of border sets. Firstly, through the principle of elitism, the top 10 performers of the current generation are copied directly into the next generation. Additionally, a novel 90 border sets that are created by recombining border sets from the current generation. Specifically, to create a new set, we select two parent border sets and, first, combine any borders that occupy similar positions across the two sets: This is done by averaging borders that are less than $5 \%$ apart between the sets (we start by combining the closest borders and the threshold is lowered when many borders are within a $5 \%$ range). Secondly, from the resulting borders, 7 are randomly selected to create a new border set. In order to converge to the optimal border-set, the selection of the two parent sets is biased towards the better performing border instantiations in the current generation as follows: $55 \%$ of the parents are selected from the best 25 networks; 30\% from the next 25 networks; and $15 \%$ of the borders are selected from the 25 networks thereafter. The bottom 25 networks (in terms of performance) do not participate in the creation of offspring. To ensure some exploration occurs, in the offspring, some borders are randomly shifted. Specifically, we randomly select $2.5 \%$ of all borders and randomly shift them (random shift is normally distributed with an SD of $2.5 \%$ of the hue spectrum). The whole process is repeated 40 times. To allow for convergence after 30 generations random mutation is switched off.

\section{Multi-Color Experiment}

\section{Software Architecture \& Stimulus}

Again, the same Resnet18 trained on ImageNet is used as in the previous experiments. In the current version the ouput layer is replaced by one with 7 output classes, each matching a category. The stimuli were designed to include 2 factors that were absent previously. Firstly, we introduce multiple, colored elements in a single stimulus: Each stimulus class is still defined by a narrow color band on the hue spectrum, but we now draw 3 words into a 224-by-224 pixel image (we will refer to these as the target words). On top of that we add 2 additional words to the image of which the color is randomly selected from the hue spectrum (we will refer to these as the distractors). All words are colored using the HSV hue spectrum at maximum brightness and saturation. Secondly, we introduce variations in color contrast. For the categorical borders to be meaningful in classifying stimuli their utility should not be overly dependent on color contrast (whether a banana is sitting on a green or a brown table, the network should still be able to use yellow to identify the banana). Therefore, we introduce a variation in color contrast by randomly selecting the color of the background from the hue spectrum. To prevent words from blending into the background 
we set the brightness of the background to $50 \%$. Stimulus examples can be found in Figure $4 \mathrm{~A}$.

\section{Procedure}

Each network is trained on colors stemming from 7 bands, each based on the borders found in the invariant border experiment. The bands, making up $10 \%$ of each category range, are shifted from the left category border to the right border in 10 steps, for each step a novel classifier is trained 15 times to obtain a reliable average. After being trained on the borders we evaluate performance for each network: The error rate for the color bands networks were trained on is obtained by evaluating those networks for ticks on the hue spectrum that fall in that range. Subsequently, we plot the error rates for the different networks as a single-colored line (see Figure $4 \mathrm{~B}$ ), to demonstrate how performance varies, depending on training bands.

\section{Objects Experiment}

\section{Software architecture and Stimulus}

Stimuli are generated using the line drawings available from the google doodle dataset ( $\mathrm{Ha}$ \& Eck, 2017). The database contains hundreds of objects, we selected a subset based on two criteria. First, we selected objects that lend itself to a simple color filling algorithm. This mainly consisted of finding objects that had clear outer borders with large spaces on the inside. Secondly, we prioritized objects that were reasonably consistent in regard to shape. The drawings are created by many different users, and, therefore, the approach could vary significantly. For instance, a user could have chosen to just draw only a cat's head prominently, or include its entire body. Of course, such variations are not unlike the variation encountered in the images the network has originally been trained on. However, we are only retraining the output layer of the network. The latter means that the network has to rely on previously trained kernels to classify the shape, and a high degree of variation may not be easy to code for.

The drawing of the stimuli follows a simple procedure. Based on the drawn lines we create a color mask. This mask is created by determining for each pixel whether it is "enclosed" by lines. Enclosed, here, is defined by having a drawn line to its left (not necessarily directly adjacent), a draw line above it, to its right and below it. After the colored area is drawn, we draw, on top of it, the drawn lines at a thickness of 4 pixels. Example results of the process can be found in Figure 5A.

\section{Procedure}

The network is trained to classify a set of 14 objects (strawberry, apple, crab, dog, school bus, cow, dolphin, mushroom, bird, submarine, angel, sweater, sailboat, duck). For the training of the network, we selected 500 samples for each object from the google doodle dataset. We also selected another 50 samples per object to have a validation set to monitor the performance of the network throughout training. The fill color for all of these objects is randomly (uniformly) selected from a narrow band on the hue spectrum. Bands are selected to be non-overlapping and having 2 bands per category, one positioned right of the category center and the other left (each takes up 1/5 of the category bounds, one in the center of the right half and one in the center of the left half of the category). This results in 14 bands that 
are not always evenly spaced, or uniformly distributed throughout the spectrum. The bands can be observed in Figure 5B.

After having trained the network, we evaluate the network using a separate set of 80 objects. Systematically changing the color of each of these 80 objects in 100 steps over the hue spectrum creates 8000 colored samples, that allow us to evaluate to what extent the object is classified based on its color.

\section{Acknowledgements}

This work was funded by the Deutsche Forschungsgemeinschaft (DFG, German Research Foundation) - project number 222641018 - SFB/TRR 135 TP C2

\section{References}

Brouwer, G. J., \& Heeger, D. J. (2013). Categorical clustering of the neural representation of color. Journal of Neuroscience, 33(39), 15454-15465. https://doi.org/10.1523/JNEUROSCI.2472-13.2013

Caves, E. M., Green, P. A., Zipple, M. N., Peters, S., Johnsen, S., \& Nowicki, S. (2018). Categorical perception of colour signals in a songbird. Nature, 560(7718), 365-367. https://doi.org/10.1038/s41586-018-0377-7

Chaabouni, R., Kharitonov, E., Dupoux, E., \& Baroni, M. (2021). Communicating artificial neural networks develop efficient color-naming systems. Proceedings of the National Academy of Sciences of the United States of America, 118(12), 1-7. https://doi.org/10.1073/pnas.2016569118

Clark, A. (2015). Pillow (PIL Fork) Documentation. readthedocs. Retrieved from https://buildmedia.readthedocs.org/media/pdf/pillow/latest/pillow.pdf

Conway, B. R., Ratnasingam, S., Jara-Ettinger, J., Futrell, R., \& Gibson, E. (2020). Communication efficiency of color naming across languages provides a new framework for the evolution of color terms. Cognition, 195(January 2019), 104086. https://doi.org/10.1016/j.cognition.2019.104086

Davidoff, J. (2001). Language and perceptual cateogrisation. Proceedings of the National Academy of Sciences of the United States of America, 74(290), 515-534. https://doi.org/10.1017/s0031819199000650

Engilberge, M., Collins, E., \& Susstrunk, S. (2017). Color representation in deep neural networks, 2786-2790.

Fagot, J., Goldstein, J., Davidoff, J., \& Pickering, A. (2006). Cross-species differences in color categorization. Psychonomic Bulletin and Review, 13(2), 275-280. https://doi.org/10.3758/BF03193843

Flachot, A., Akbarinia, A., Schütt, H. H., Fleming, R. W., Wichmann, F. A., \& Gegenfurtner, K. R. (2020). Deep Neural Models for color discrimination and color constancy, 1-19. Retrieved from http://arxiv.org/abs/2012.14402

Flachot, A., \& Gegenfurtner, K. R. (2018). Processing of chromatic information in a deep convolutional neural network. Journal of the Optical Society of America A, 35(4), B334. https://doi.org/10.1364/josaa.35.00b334

Gibson, E., Futrell, R., Jara-Ettinger, J., Mahowald, K., Bergen, L., Ratnasingam, S., ... Conway, B. R. (2017). Color naming across languages reflects color use. Proceedings of the National Academy of Sciences of the United States of America, 114(40), 10785-10790. 
https://doi.org/10.1073/pnas.1619666114

Goldstone, R. L., \& Hendrickson, A. T. (2010). Categorical perception. Wiley Interdisciplinary

Reviews: Cognitive Science, 1(1), 69-78. https://doi.org/10.1002/wcs.26

Ha, D., \& Eck, D. (2017). A neural representation of sketch drawings. ArXiv.

Hanley, D., Grim, T., Igic, B., Samaš, P., López, A. V., Shawkey, M. D., \& Hauber, M. E. (2017).

Egg discrimination along a gradient of natural variation in eggshell coloration. Proceedings of the Royal Society B: Biological Sciences, 284(1848). https://doi.org/10.1098/rspb.2016.2592

Hansen, T., \& Gegenfurtner, K. R. (2017). Color contributes to object-contour perception in natural scenes. Journal of Vision, 17(3), 1-19. https://doi.org/10.1167/17.3.14

Harnad, S. (1987). Categorical Perception: The groundwork of cognition. New York, NY, USA: Cambridge University Press.

He, K., Zhang, X., Ren, S., \& Sun, J. (2016). Deep residual learning for image recognition. Proceedings of the IEEE Computer Society Conference on Computer Vision and Pattern Recognition, 2016-Decem, 770-778. https://doi.org/10.1109/CVPR.2016.90

Jia Deng, Wei Dong, Socher, R., Li-Jia Li, Kai Li, \& Li Fei-Fei. (2009). ImageNet: A large-scale hierarchical image database, 248-255. https://doi.org/10.1109/cvprw.2009.5206848

Jones, C. D., Osorio, D., \& Baddeley, R. J. (2001). Colour categorization by domestic chicks. Proceedings of the Royal Society B: Biological Sciences, 268(1481), 2077-2084. https://doi.org/10.1098/rspb.2001.1734

Jraissati, Y. (2014). Proving universalism wrong does not prove relativism right: Considerations on the ongoing color categorization debate. Philosophical Psychology, 27(3), 401-424. https://doi.org/10.1080/09515089.2012.733815

Kastak, D., \& Schusterman, R. J. (1994). Transfer of visual identity matching-to-sample in two california sea lions (zalophus californianus). Animal Learning \& Behavior, 22(4), 427-435. https://doi.org/10.3758/BF03209162

Kay, P., \& Regier, T. (2006). Language, thought and color: recent developments. Trends in Cognitive Sciences, 10(2), 51-54. https://doi.org/10.1016/j.tics.2005.12.003

Kay, P. (2015). Universality of Color Categorization. In A. J. Elliot, M. D. Fairchild, \& A. Franklin (Eds.), Handbook of color psychology (pp. 245-258). Cambridge University Press.

Kay, P., \& Kempton, W. (1984). What Is the Sapir-Whorf Hypothesis?? American Anthropologist.

Kay, P., \& Regier, T. (2003). Resolving the question of color naming universals. Proceedings of the National Academy of Sciences of the United States of America, 100(15), 90859089. https://doi.org/10.1073/pnas.1532837100

Kay, P., Regier, T., Gilbert, A. L., \& Ivry, R. B. (2009). Lateralized Whorf: Language influences perceptual decision in the right visual field. Language Evolution and the Brain, (2007), 261-284.

Kiper, D. C., Fenstemaker, S. B., \& Gegenfurtner, K. R. (1997). Chromatic properties of neurons in macaque area V2. Visual Neuroscience, 14(6), 1061-1072. https://doi.org/10.1017/s0952523800011779

Lennie, P., Krauskopf, J., \& Sclar, G. (1990). Chromatic mechanisms in striate cortex of macaque. Journal of Neuroscience, 10(2), 649-669. https://doi.org/10.1523/jneurosci.10-02-00649.1990

Lindsey, D. T., Brown, A. M., Reijnen, E., Rich, A. N., Kuzmova, Y. I., \& Wolfe, J. M. (2010). Color channels, not color appearance or color categories, guide visual search for 
desaturated color targets. Psychological Science, 21(9), 1208-1214. https://doi.org/10.1177/0956797610379861

Marcel, S., \& Rodriguez, Y. (2010). Torchvision the Machine-Vision Package of Torch. In Proceedings of the 18th ACM International Conference on Multimedia. New York, NY, USA: Association for Computing Machinery. https://doi.org/10.1145/1873951.1874254

Poralla, J., \& Neumeyer, C. (2006). Generalization and categorization of spectral colors in goldfish. II. Experiments with two and six training wavelengths. Journal of Comparative Physiology A: Neuroethology, Sensory, Neural, and Behavioral Physiology, 192(5), 469479. https://doi.org/10.1007/s00359-005-0082-5

Rafegas, I., \& Vanrell, M. (2018). Color encoding in biologically-inspired convolutional neural networks. Vision Research, 151(February 2017), 7-17. https://doi.org/10.1016/j.visres.2018.03.010

Ray, V. F. (1952). Techniques and Problems in the Study of Human Color Perception. Southwestern Journal of Anthropology, 8(3), 251-259.

Regier, T., Kay, P., \& Cook, R. S. (2005). Focal colors are universal after all. Proceedings of the National Academy of Sciences of the United States of America, 102(23), 8386-8391. https://doi.org/10.1073/pnas.0503281102

Roberson, D., Davidoff, J., \& Braisby, N. (1999). Similarity and categorisation: Neuropsychological evidence for a dissociation in explicit categorisation tasks. Cognition, 71(1), 1-42. https://doi.org/10.1016/S0010-0277(99)00013-X

Roberson, D., Davidoff, J., \& Davies, I. (2000). Color categories are not universal: Replications and new evidence from a stone-age culture. Journal of Experimental Psychology: General, 129(3), 369-398. https://doi.org/10.1037/0096-3445.129.3.369

Roberson, D., Davidoff, J., Davies, I. R. L., \& Shapiro, L. R. (2005). Color categories: Evidence for the cultural relativity hypothesis. Cognitive Psychology, 50(4), 378-411. https://doi.org/10.1016/j.cogpsych.2004.10.001

Roberson, D., Davies, I. R. L., Davidoff, J., \& Shapiro, L. R. (2004). The development of color categories in two languages: A longitudinal study. Journal of Experimental Psychology: General, 133(4), 554-571. https://doi.org/10.1037/0096-3445.133.4.554

Rosenthal, I., Ratnasingam, S., Haile, T., Eastman, S., Fuller-Deets, J., \& Conway, B. R. (2018). Color statistics of objects, and color tuning of object cortex in macaque monkey. Journal of Vision, 18(11), 1-21. https://doi.org/10.1167/18.11.1

Siuda-Krzywicka, K., Witzel, C., Chabani, E., Taga, M., Coste, C., Cools, N., ... Bartolomeo, P. (2019). Color Categorization Independent of Color Naming. Cell Reports, 28(10), 24712479.e5. https://doi.org/10.1016/j.celrep.2019.08.003

Siuda-Krzywicka, K., Witzel, C., Taga, M., Delanoe, M., Cohen, L., \& Bartolomeo, P. (2020). When colours split from objects: The disconnection of colour perception from colour language and colour knowledge. Cognitive Neuropsychology, 37(5-6), 325-339. https://doi.org/10.1080/02643294.2019.1642861

Skelton, A. E., Catchpole, G., Abbott, J. T., Bosten, J. M., \& Franklin, A. (2017). Biological origins of color categorization. Proceedings of the National Academy of Sciences of the United States of America, 114(21), 5545-5550. https://doi.org/10.1073/pnas.1612881114

Skinner, B. F. (1950). Are theories of learning necessary? Psychological Review, 57(4), 193216. https://doi.org/10.1037/h0054367

Steels, L., \& Belpaeme, T. (2005). Coordinating perceptually grounded categories through language: A case study for colour. Behavioral and Brain Sciences, 28(4), 469-489. 
https://doi.org/10.1017/S0140525X05000087

Twomey, C. R., Roberts, G., Brainard, D., \& Plotkin, J. B. (2020). What we talk about when we talk about color. BioRxiv. https://doi.org/10.1101/2020.09.29.319517

Winawer, J., Witthoft, N., Frank, M. C., Wu, L., Wade, A. R., \& Boroditsky, L. (2007). Russian blues reveal effects of language on color discrimination. Proceedings of the National Academy of Sciences of the United States of America, 104(19), 7780-7785. https://doi.org/10.1073/pnas.0701644104

Witzel, C., \& Gegenfurtner, K. R. (2011). Is there a lateralized category effect for color? Journal of Vision, 11(12), 1-25. https://doi.org/10.1167/11.12.16

Witzel, C., \& Gegenfurtner, K. R. (2013). Categorical sensitivity to color differences. Journal of Vision, 13(7), 1-33. https://doi.org/10.1167/13.7.1

Witzel, C., \& Gegenfurtner, K. R. (2018). Color Perception: Objects, Constancy, and Categories. Annual Review of Vision Science, 475-499.

Wright, A. A., \& Cumming, W. W. (1971). Color-Naming Functions for the Pigeon. Training, (I), 7-17.

Yendrikhovskij, S. N. (2001). A Computational Model of Colour Categorization. In Color Research \& Application: Endorsed by Inter-Society Color Council, The Colour Group (Great Britain), Canadian Society for Color, Color Science Association of Japan, Dutch Society for the Study of Color, The Swedish Colour Centre Foundation, Colour Soc (Vol. 26.S1).

Zaidi, Q., \& Conway, B. (2019). Steps towards neural decoding of colors. Current Opinion in Behavioral Sciences, 30, 169-177. https://doi.org/10.1016/j.cobeha.2019.10.011

Zaslavsky, N., Kemp, C., Regier, T., \& Tishby, N. (2018). Efficient compression in color naming and its evolution. Proceedings of the National Academy of Sciences of the United States of America, 115(31), 7937-7942. https://doi.org/10.1073/pnas.1800521115

Zaslavsky, N., Kemp, C., Tishby, N., \& Regier, T. (2019). Color Naming Reflects Both Perceptual Structure and Communicative Need. Topics in Cognitive Science, 11(1), 207219. https://doi.org/10.1111/tops.12395

Zaslavsky, N., Kemp, C., Tishby, N., \& Regier, T. (2020). Communicative need in colour naming. Cognitive Neuropsychology, 37(5-6), 312-324. https://doi.org/10.1080/02643294.2019.1604502 